\title{
Principal component analysis reveals the 1000 Genomes Project does not sufficiently cover the human genetic diversity in Asia
}

\section{Dongsheng Lu and Shuhua $X u^{*}$}

Max Planck Independent Research Group on Population Genomics, Chinese Academy of Sciences and Max Planck Society Partner Institute for Computational Biology, Shanghai Institutes for Biological Sciences, Chinese Academy of Sciences, Shanghai, China

\section{Edited by:}

Jianzhong Ma, University of Texas MD Anderson Cancer Center, USA

\section{Reviewed by:}

Xiangqing Sun, Case Western

Reserve University, USA

Xiaoming Liu, The University of Texas Health Science Center at Houston, USA

\section{*Correspondence:}

Shuhua Xu, Max Planck Independent Research Group on Population Genomics, Chinese Academy of Sciences and Max Planck Society Partner Institute for Computational Biology, Shanghai Institutes for Biological Sciences, Chinese

Academy of Sciences, Yueyang Road 320, Shanghai 200031, China

e-mail: xushua@picb.ac.cn
The 1000 Genomes Project ( $1 \mathrm{KG}$ ) aims to provide a comprehensive resource on human genetic variations. With an effort of sequencing 2,500 individuals, $1 \mathrm{KG}$ is expected to cover the majority of the human genetic diversities worldwide. In this study, using analysis of population structure based on genome-wide single nucleotide polymorphisms (SNPs) data, we examined and evaluated the coverage of genetic diversity of $1 \mathrm{KG}$ samples with the available genome-wide SNP data of 3,831 individuals representing 140 population samples worldwide. We developed a method to quantitatively measure and evaluate the genetic diversity revealed by population structure analysis. Our results showed that the $1 \mathrm{KG}$ does not have sufficient coverage of the human genetic diversity in Asia, especially in Southeast Asia. We suggested a good coverage of Southeast Asian populations be considered in $1 \mathrm{KG}$ or a regional effort be initialized to provide a more comprehensive characterization of the human genetic diversity in Asia, which is important for both evolutionary and medical studies in the future.

Keywords: human genetic diversity, population structure, 1000 Genomes Project, Pan-Asian SNP Project, Human Genome Diversity Project, single nucleotide polymorphisms, principal component analysis

\section{INTRODUCTION}

Due to recent burgeoning in next generation sequencing (NGS) technologies, whole-genome sequencing of a large number of individuals in multiple populations is now feasible. This advanced technology has facilitated genome-wide investigation of human genetic variations and inference of population demographic history and natural selection without ascertainment bias (Teo et al., 2010). Pioneer efforts of 1000 Genomes Project (1KG) based on NGS at low coverage have already identified millions of variants, including single nucleotide polymorphisms (SNPs), insertion/deletions (INDELs), and structural variations (SVs) in diverse populations worldwide, which has made remarkable achievements in the studies of human genetic variation, natural selection, human disease mapping, and local adaptation to environments (Zawistowski et al., 2010; Holm et al., 2011; Pasaniuc et al., 2012). Another international effort prior to $1 \mathrm{KG}$, the International HapMap Project (HapMap), investigated human common genetic variations in human populations using genotyping technology. However, both projects included only a few number of Asian population samples that were exclusively from East Asia, such as $\mathrm{CHB}$ (Han Chinese in Beijing, China), CHD (Chinese in Denver, CO, USA), and JPT (Japanese in Tokyo, Japan; The International HapMap Consortium, 2005), which could only represent a small proportion of human genetic diversity in Asia. The Human Genome Diversity Project (HGDP), one of the most widely used resources, also lacked good representation of samples especially from Southeast Asia. Although HGDP (Li et al., 2008) consists of many East Asian and some Oceanian populations such as Melanesian and Papuan, there is almost no Southeast Asian population sample except for a single Cambodian population. Although $1 \mathrm{KG}$ has newly incorporated two more Asian populations KHV (Kinh in Ho Chi Minh City, Vietnam) and CDX (Chinese Dai in Xishuangbanna) mainly from southern part of China which could be treated as Southeast Asian populations, they may only explain a very small proportion of genetic diversity in Southeast Asian populations. With the genome-wide SNP data in a large number of 47 Southeast Asian population samples investigated in the HUGO Pan-Asian SNP Project (PASNP; The HUGO Pan-Asian SNP Consortium, 2009), we have a good opportunity to examine and evaluate the coverage of human genetic diversity in 1 KG data. Based on the SNP data obtained from PASNP, $1 \mathrm{KG}$, and HGDP, we systematically investigated and evaluated whether the genetic diversity coverage in $1 \mathrm{KG}$ was sufficient to represent the high population diversity in Asia, especially in Southeast Asia considering the limited number of population samples included in 1KG. We developed a method to measure and evaluate the coverage of $1 \mathrm{KG}$ samples at a scale of Asia-Pacific region based on principal component analysis (PCA) of genome-wide SNP data. Our results have many implications for further study design and provide some directions for international or regional collaborative efforts to investigate human variants. 


\section{MATERIALS AND METHODS POPULATIONS AND SAMPLES}

We analyzed the latest release of the data (version 3 of phase 1, March 2012 release) of the $1 \mathrm{KG}$ and fetched around $36.5 \mathrm{M}$ autosomal SNPs of 1,092 individuals representing 14 populations worldwide (The 1000 Genomes Project Consortium, 2010). The genotypes of 938 unrelated individuals representing 53 populations were obtained from the Human Genome Diversity Panel-CEPH (HGDP-CEPH; Rosenberg et al., 2002; Li et al., 2008). The Illumina $650 \mathrm{~K}$ annotation file with genome version GRCh37 downloaded from UCSC ${ }^{1}$ was used for the coordinates of HGDP dataset to facilitate the combination. For PASNP, we used the Affymetrix GeneChip Human Mapping 50K Xba array annotation file with netaffx-build 32 (GRCh37) from Affymetrix website to call the SNPs from raw CEL files, followed by quality control using the same criteria as used in the original paper (The HUGO Pan-Asian SNP Consortium, 2009). Approximately 57,323 autosomal SNPs were identified from 1,713 individuals representing 71 populations mainly from Southeast Asia and East Asia.

As the variants of individuals from two Asian populations, CDX and KHV, from $1 \mathrm{KG}$ had not been released when we initiated this study, we also incorporated the BAM (binary version of Sequence Alignment/Map format) files of 95 individuals from CDX and 49 individuals from KHV downloaded from $1 \mathrm{KG}$ website.

\section{VARIANTS CALLING FOR CDX AND KHV POPULATIONS}

The well-curated BAM files from CDX (95) and KHV (49) were processed using the GATK (McKenna etal., 2010; GATK-1.22-g8143) to call the raw variants, followed by variant quality recalibration (Depristo et al., 2011) of the default pipeline of next generation sequence data SNP calling at the Broad Institute. Around 16.9M SNPs were identified by joint calling across all samples from CDX and KHV. Then the SNP loci with QUAL value no less than 100 and individual SNP loci with DP (single locus sequencing depth) no less than 4 were applied to keep the high-quality SNPs for each individual. Then the individuals with missing data larger than $30 \%$, or loci with missing genotypes larger than $20 \%$ in any of the three databases (1KG, HGDP, and PASNP) were excluded. The remaining 18,874 SNPs of 60 individuals from CDX and 43 from KHV were used for the data combination.

\section{COMBINED GENOTYPE DATA}

As the datasets were from sequencing or genotyping on different platforms, we identified a set of intersected SNPs among these three datasets and used only data from autosomal chromosomes. Any SNP with discordant annotation among any pair of populations is not included in the combined genotype data, for example, strand bias (SNPs with $\mathrm{A} / \mathrm{G}$ in PASNP and $\mathrm{C} / \mathrm{G}$ in $1 \mathrm{KG}$, or SNPs with $\mathrm{A} / \mathrm{T}$ in $\mathrm{HGDP}$ but $\mathrm{A} / \mathrm{C}$ in $1 \mathrm{KG}$ ) and annotation misleading (rs1611684 of chr6:29825846 in HGDP is A/C, but the individual genotype is AA, AG, or GG). Next, the SNPs with missing genotypes larger than $20 \%$ in a population were discarded. Finally, we

\footnotetext{
${ }^{1}$ http://hgdownload.cse.ucsc.edu/goldenPath/hg19/database/
}

identified a set of 7,775 clean autosomal SNPs from 3,832 individuals in these three datasets. We noted that the number of SNPs was smaller than the maximum set of overlapping SNPs shared among these datasets, as some SNPs were filtered out during the quality control procedures.

\section{GEOGRAPHIC LOCATION CLASSIFICATION}

We divided the original geographic location of each population in the combined data into eight main areas, i.e., Africa, Native America, Europe, West Asia, Central and South Asia, East Asia, Southeast Asia, and Oceania (Table 1). The original geographic location of each population was obtained from the publication of each dataset (Figure 1). Considering the great influence of population admixture and complex human migration history in Southeast Asia as well as the substantial linguistic diversity, we used the linguistic affiliations mentioned in the original publication (The HUGO Pan-Asian SNP Consortium, 2009) to facilitate the classification. For example, the TH-HM population living in Thailand is of Hmong-Mien, Hmongic, Chuanqiandian linguistic affiliation, which has the same linguistic affiliation as that of CNHM population from Guizhou, China. Therefore, the TH-HM population was considered as an East Asian population. Due to the fact that Yunnan in China is adjacent to Southeast Asia and the possible migration among these regions, CDX and KHV populations are considered as Southeast Asian populations. To compare the population genetic diversity difference between Southeast Asia and East Asia, we classified the populations in PASNP into nine linguistic groups as mentioned in the original paper, i.e., Altaic (JP-ML, JP-RK, KR-KR, CN-UG), Sino-Tibetan (SG-CH, THKA, TW-HA, TW-HB, CN-GA, CN-JN, IN-TB), Hmong-Mien (TH-HM, TH-YA, CN-HM), Tai-Kadai (TH-TK, TH-TL, THTU, TH-TY, CN-CC), Austro-Asiatic (MY-JH, MY-KS, TH-LW, TH-MA, TH-MO, TH-PL, TH-PP, TH-TN, CN-WM, CN-WU), Papuan (AX-ME), Dravidian (SG-ID, IN-DR), Indo-European (IN-EL, IN-IL, IN-NI, IN-NL, IN-SP, IN-WI, IN-WL), with the rest populations all belonging to Austronesian.

\section{PRINCIPAL COMPONENT ANALYSIS}

Principal component analysis was performed with EIGENSOFT version 3.0 (Price et al., 2006) at the individual level across different geographic scales from worldwide to Asia-pacific region. We used an in-house $\mathrm{R}^{2}$ script to facilitate the PCA figure plotting. The proportions of variance explained by each principal component were shown in the PCA plot. The top two principal components PC1 and PC2 were chosen for the population genetic diversity comparison, so that each individual was represented by a point in PCA plot.

\section{A STATISTIC TO MEASURE GENETIC DIVERSITY COVERAGE}

The assumption of our approach was that the genetic diversity of a certain population or region could be measured by the representation of individuals in a two-dimensional space defined by a number of PCs, for instance, PC1 and PC2. To quantitatively evaluate the genetic diversity in Asian populations between two different datasets, such as $1 \mathrm{KG}$ and another dataset, we defined $D_{d}$

\footnotetext{
${ }^{2}$ www.r-project.org
} 
Table 1 | The population identities from 1KG, HGDP, and PASNP for each one of the eight main areas.

\begin{tabular}{|c|c|c|c|}
\hline Region & $1 K G$ & PASNP & HGDP \\
\hline Africa & YRI, LWK, ASW & & $\begin{array}{l}\text { Bantu Kenya, Bantu South Africa, Mandenka, } \\
\text { Yoruba, San, MbutiPygmy, Biaka, Mozabite }\end{array}$ \\
\hline Native America & $M X L, P U R, C L M$ & $A X-A l$ & Karitiana, Surui, Colombian, Maya, Pima \\
\hline Europe & CEU, TSI, GBR, FIN, IBS & & $\begin{array}{l}\text { Orcadian, Adygei, Russian, Basque, French, Italian, } \\
\text { Sardinian, Tuscan }\end{array}$ \\
\hline West Asia & & & Bedouin, Druze, Palestinian \\
\hline Central and South Asia & & CN-UG, IN-* SG-ID & $\begin{array}{l}\text { Balochi, Brahui, Makrani, Sindhi, Pathan, Burusho, } \\
\text { Hazara, Uyghur, Kalash, Xibo }\end{array}$ \\
\hline East Asia & $\mathrm{CHB}, \mathrm{CHS}, \mathrm{JPT}$ & $\begin{array}{l}\text { KR-KR, JP-ML, JP-RK, TW-HA, TW-HB, AX-AT, } \\
\text { AX-AM, CN-GA, CN-HM, TH-HM, SG-CH }\end{array}$ & $\begin{array}{l}\text { Han-N China, Han, Daur, Hezhen, Miao, Oroqen, } \\
\text { She, Tu, Yi, Mongolia, Japanese, Yakut }\end{array}$ \\
\hline Southeast Asia & CDX, KHV & $\begin{array}{l}\text { SG-MY, CN-CC, CN-WA, CN-SH, CN-JN, TH-* } \\
\text { ID-* }{ }^{*} \mathrm{Pl}_{-*}^{*}, \mathrm{MY}^{*}\end{array}$ & Dai, Lahu, Tujia, Naxi, Cambodian \\
\hline Oceania & & AX-ME & Melanesian, Papuan \\
\hline
\end{tabular}

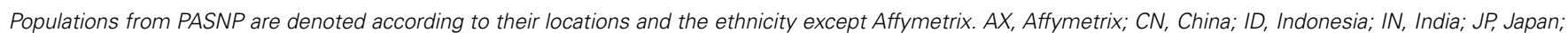

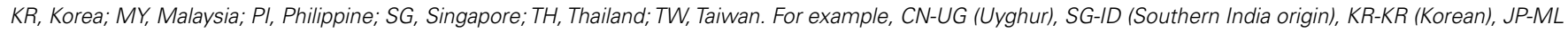

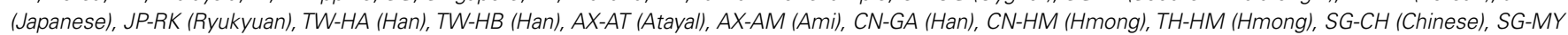

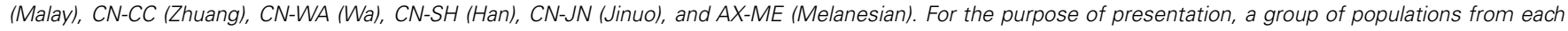

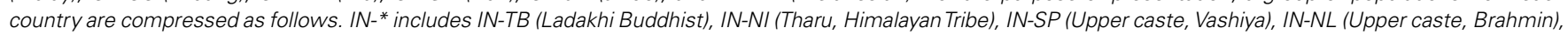

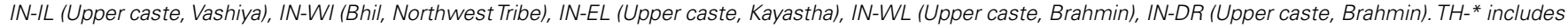

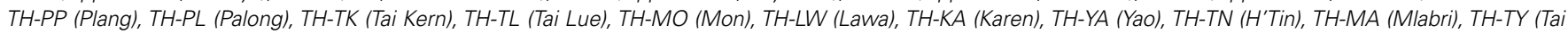

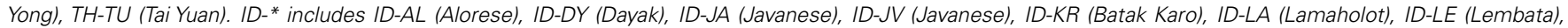

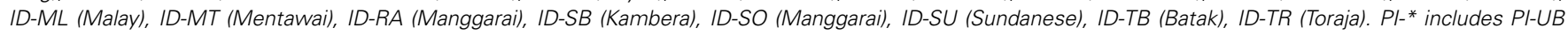

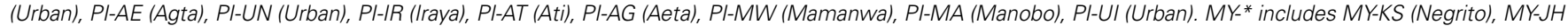
(Negrito), MY-KN (Malay), MY-TM (Proto-Malay), MY-MN (Malay), MY-BD (Bidayuh).

as follows:

$$
D_{d}=\log \frac{\sum_{s=1}^{S} \delta_{P\left(K_{1 i}, K_{2 i}\right) \in R_{s}\left(d_{1}, d_{2}\right)}}{\sum_{s=1}^{S} \delta_{P\left(O_{1 j}, O_{2 j}\right) \in R_{s}\left(d_{1}, d_{2}\right)}},
$$

where $P\left(K_{1 i}, K_{2 i}\right)$ denotes one point $\left(\mathrm{PC} 1=K_{1 i}, \mathrm{PC} 2=K_{2 i}\right)$ in PC plot representing individual $i$ from $1 \mathrm{KG}$, while $P\left(O_{1 i}, O_{2 i}\right)$ represents individual $j$ from HGDP and PASNP. The PC plot can be divided into $S$ rectangles by a grid with side-length $d_{1}$ and $d_{2} . \delta_{P\left(K_{1}, K_{2 i}\right) \in R_{s}\left(d_{1}, d_{2}\right)}$ was counted once if there is any individual located in the rectangle defined by $R_{s}\left(d_{1}, d_{2}\right)$, otherwise 0 if there is no individual in it. The number of rectangles in the grid overlapping with the points representing the individuals from $1 \mathrm{KG}, \mathrm{HGDP}$, and PASNP can be an estimation of the coverage for $1 \mathrm{KG}$, and HGDP and PASNP, respectively. Next we defined the logarithm of the ratio to the base $e$ as $D_{d}$. The difficult part of $D_{d}$ is how to assign $d_{1}$ and $d_{2}$ to an optimal estimation of the different coverage for the population pairs, which depends on the average distance $\overline{d_{1}}$ and $\overline{d_{2}}$ of the projection of individuals on PC1 and PC2, respectively. We further introduced a coefficient $m$ and defined $d_{1}=m \overline{d_{1}}$ and $d_{2}=m \overline{d_{2}}$, where $m=1,2,3, \ldots$..

\section{RESULTS PRINCIPAL COMPONENT ANALYSIS SHOWING UNDER-REPRESENTATION OF SOUTHEAST ASIAN GENETIC DIVERSITY IN 1KG}

We performed PCA with EIGENSOFT version 3.0 (Price et al., 2006) for all the 3,813 available individuals from 140 populations worldwide, and a two-dimensional plot based on the top two PCs was shown in Figure 2A. As expected, individuals from Asia, Europe, and Africa can be well distinguished. The overrepresenting of East Asian and Southeast Asian samples from HGDP and PASNP than those from $1 \mathrm{KG}$ was due to the larger sample size in HGDP and PASNP, so we randomly selected individuals from each geographical area to match sample size so that our results are not biased by the fluctuation of sample size in different regions. As there were no West Asian, Central and South Asian, and Oceanian individuals in $1 \mathrm{KG}$ phase I, we removed individuals from those regions in HGDP and PASNP and did PCA again (Figure 2B). The result showed that $1 \mathrm{KG}$ has lower coverage in East Asia and Southeast Asia compared to HGDP and PASNP, but reasonable coverage in America. For example, $1 \mathrm{KG}$ does not have any representation for some Asian populations such as Yakut (empty triangles in sea green) and Pacific islanders (green crosses) from Southeast Asia. 

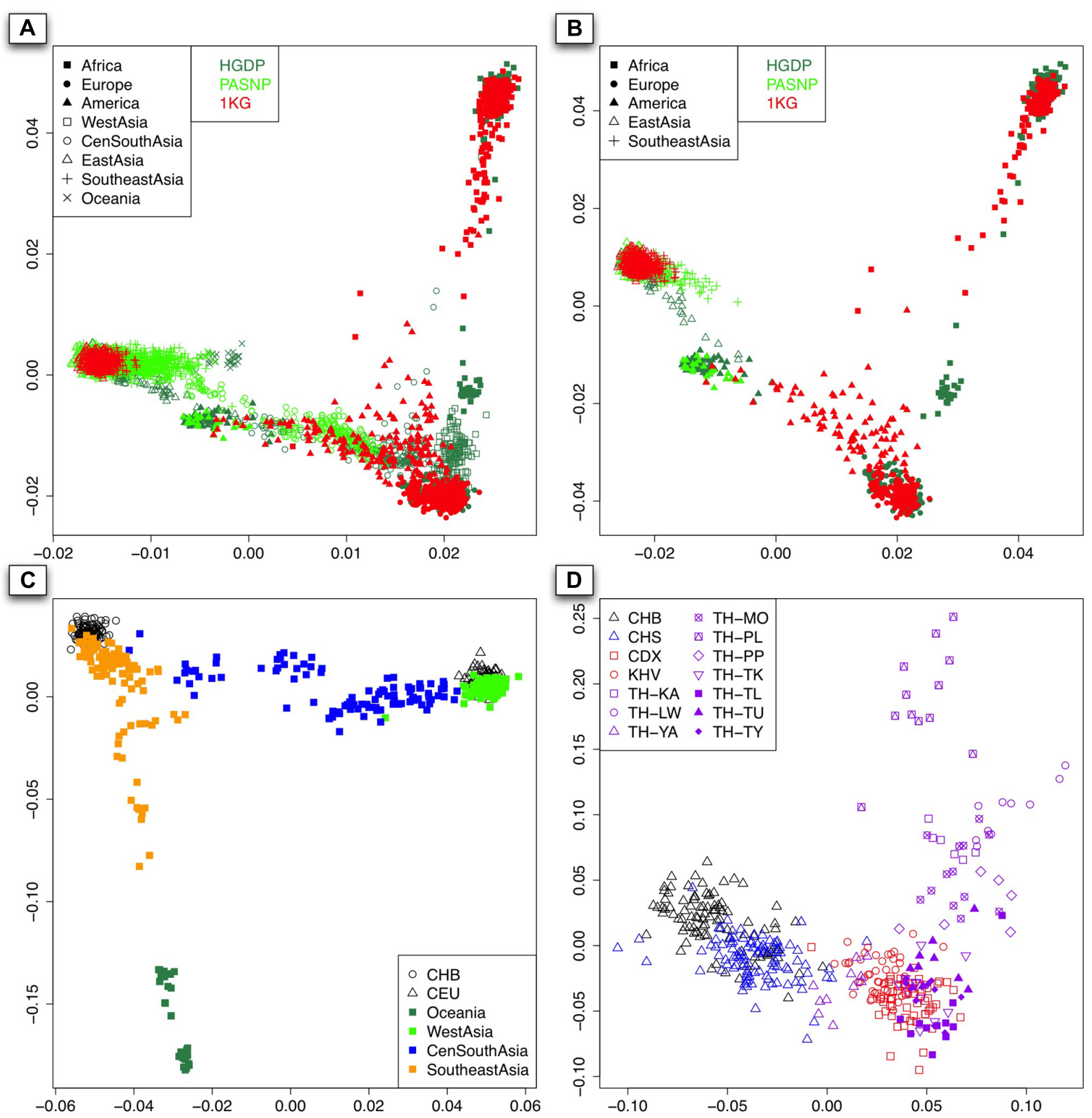

FIGURE 2 | (A) The PCA result of all the 140 populations worldwide from $1 \mathrm{KG}$, HGDP, and PASNP without sampling. $x$-Axis denotes the value of PC1, while $y$-axis denotes the value of PC2, with each dot in the figure representing one individual. The color for individuals from $1 \mathrm{KG}, \mathrm{HGDP}$, and PASNP are red, sea green, and green, respectively. (B) The first two components of PCA result

based on randomly selected individuals belonging to Africa, Europe, America,

We were also interested in evaluating whether $1 \mathrm{KG}$ has sufficient diversity coverage in different scales besides worldwide samples comparison. We used similar sampling approach as described above and pooled population samples from East Asia, Southeast Asia, and Oceania (Figure 3B). We examined distribution of $1 \mathrm{KG}$ samples in the PC plots in the context including both Southeast Asian and East Asian populations (Figure 3C), and also in the context including only Southeast Asian populations (Figure 3D).
East Asia, and Southeast Asia in the $1 \mathrm{KG}$ or HGDP and PASNP have larger sample size in the corresponding geographic area. The colors for each dataset are the same as (A). (C). The PCA result using individuals from $\mathrm{CHB}, \mathrm{CEU}$, Oceania, West Asia, Central and South Asia (CenSouthAsia), and Southeast Asia. (D). The PCA result when pooling individuals from $\mathrm{CHB}, \mathrm{CHS}, \mathrm{CDX}, \mathrm{KHV}$, and individuals from Thailand except for TH-MA and TH-TN. 

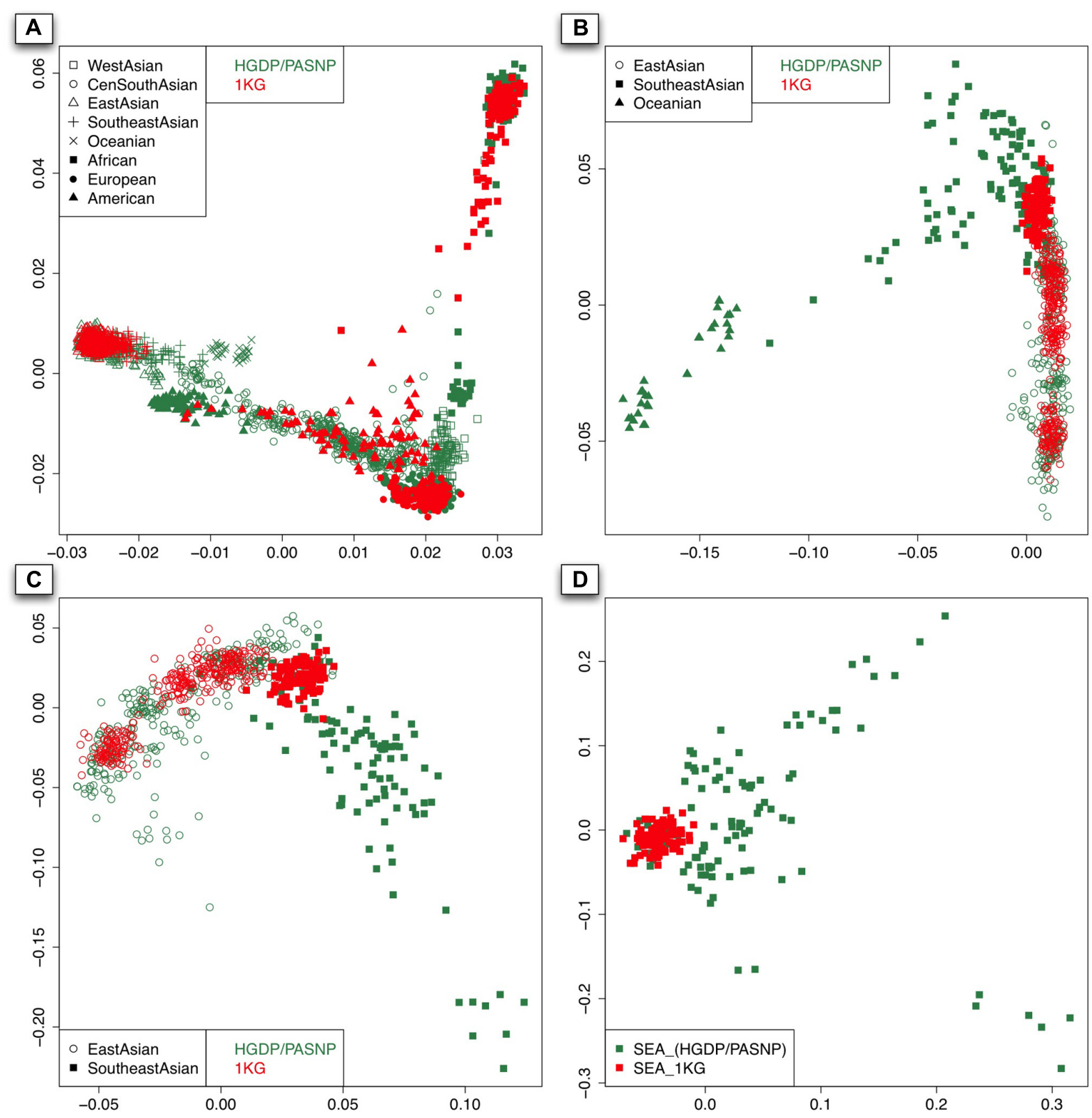

D

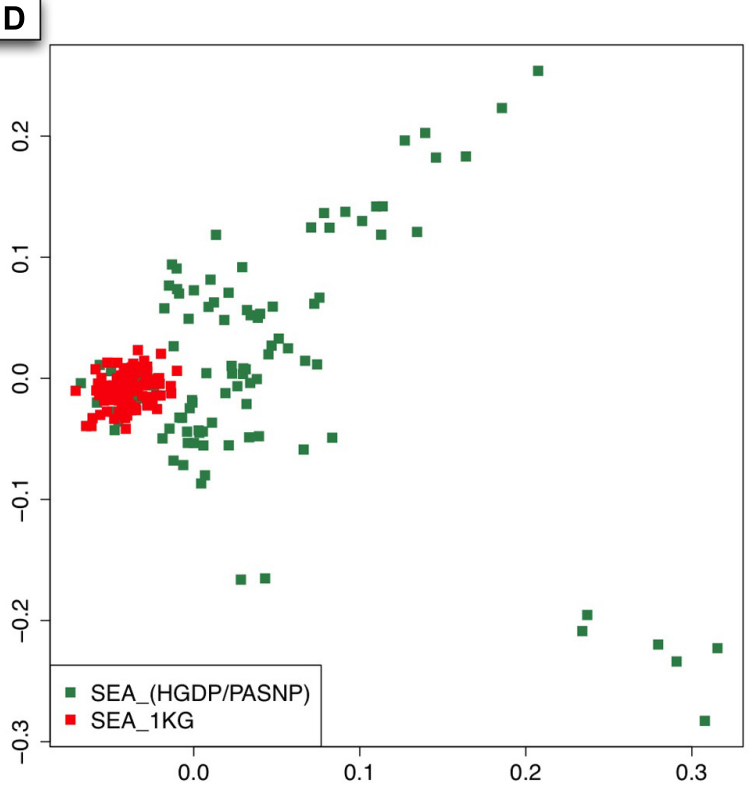

FIGURE 3 |The first two components of the PCA result with sampling approach based on different contexts: worldwide population context (A). East Asian and Pacific islander context (B). East Asian and Southeast Asian

contexts (C). and Southeast Asian context (D). The individuals with red color are from $1 \mathrm{KG}$ and those with sea green color are from the other two datasets.

of population samples based on PCA results. To systematically measure such representation in different contexts, we examined eight more groups based on their geographical distributions of populations: non-Africans (NA), Eurasians and Oceanians (EAO), Asians and Oceanians (AO), non-Western Asians and Oceanians (NWAO), East Asians and Pacific islanders (EPI), East Asians and Southeast Asians (ESEA), Southeast Asians and Oceanians (SEAO), and Southeast Asians (SEA). Again, we applied similar sampling procedure to reduce the effect of unbalanced sample sizes between $1 \mathrm{KG}$ and the control groups.
To investigate the influence of $m$, which can be treated as a coefficient of $\overline{d_{1}}\left(d_{1}=m \overline{d_{1}}\right)$ and $\overline{d_{2}}\left(d_{2}=m \overline{d_{2}}\right)$ used to define the size of rectangle in the grid of the PCs (see Materials and Methods), we set $D_{d}$ as a function of $m$ and displayed the relationship of $m$ and $D_{d}$ in Figure 4 . In the context of worldwide populations, $D_{d}$ for Southeast Asians went down steeply while $m$ increased from 1 to 20 (Figure 4A) and showed considerable fluctuations subsequently beyond 20 . To illustrate the performance of $D_{d}$ in the situation of good representation of population samples, we evaluated coverage of $1 \mathrm{KG}$ in European populations using $D_{d}$ based on HGDP and PASNP data. The 


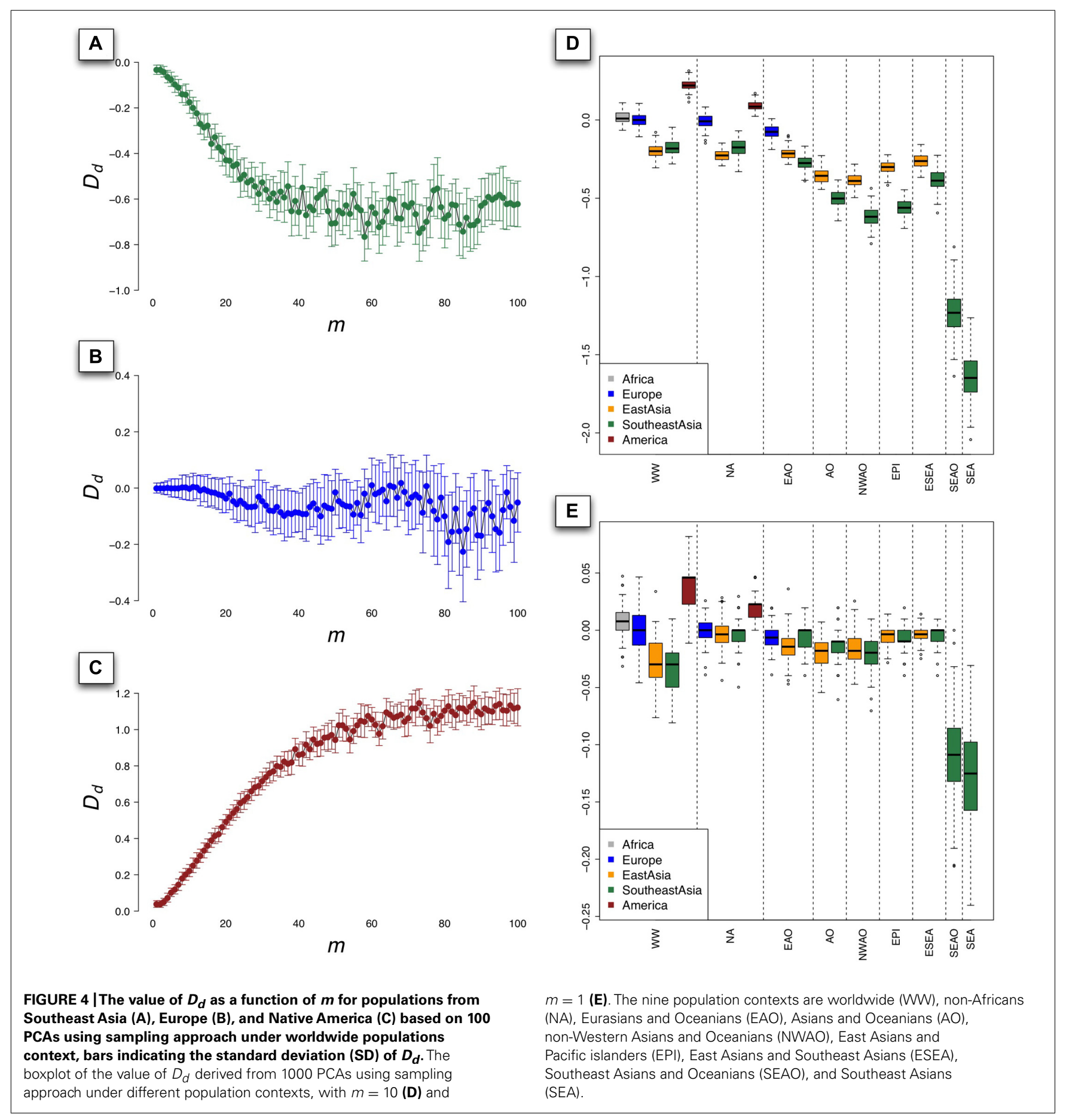

results showed that $D_{d}$ did not deviate significantly from 0 in the context of European populations, even with different values of $m$ (Figure 4B). Interestingly, the $D_{d}$ values have quite different behavior in the context of Native Americans, which were larger than 0 and positively correlated with $m$ (Figure 4C). This pattern indicated that Native American samples are overrepresented in $1 \mathrm{KG}$ compared with those in HGDP and PASNP data, which were also supported by the result illustrated by Figure 2B. Since our method was based on PCA results and the

individuals with similar coordinates in PC plots are likely to be genetically closely related, we chose only one individual as proxy of diversity in the area defined by $m$ to reduce redundancy. We suggested that the value of $m$ should be neither too small nor too large. Especially, $m=1$ does not make any sense since in this case any two individuals would not be likely to be in the same rectangle defined by $R_{s}\left(d_{1}, d_{2}\right)$. When $m$ was given a very large value, the probability of two distantly related individuals from different populations to be located in the same rectangle 
would be very high. In both cases, it would lead to unreasonable estimations.

To evaluate genetic diversity coverage of $1 \mathrm{KG}$ in a specific geographic area, we calculated $D_{d}$ with the sampling procedure repeated for 100 times using $m=10$. The results of $D_{d}$ for Native American populations based on worldwide population context $(\mathrm{WW})$ and NA context were all significantly greater than 0 $\left(p=2 \times 10^{-84}\right.$ and $4 \times 10^{-50}$, Student's $t$-test; Figure 4D), which indicated high genetic diversity coverage of $1 \mathrm{KG}$ in this area. The values of $D_{d}$ for European populations under WW context and NA context did not significantly deviate from $0(p=0.66$ and 0.13$)$, but less than 0 based on EAO context $\left(p=5.2 \times 10^{-32}\right)$. However, the values of $D_{d}$ for East Asian populations were all significantly less than $0\left(p<7 \times 10^{-64}\right)$, even if we set $m=1$ in the calculation ( $p=0.006$ for NA and $p<10^{-6}$ for other contexts; Figure 4E). The results for Southeast Asian populations based on AO, NWAO, EPI still had lower values of $D_{d}$ than those for East Asian populations, which indicated that the genetic diversity coverage of $1 \mathrm{KG}$ in Southeast Asia was much less sufficient. As we looked closer, the only two population samples (KHV and CDX) collected from Southeast Asia in $1 \mathrm{KG}$ could represent a small proportion of genetic diversity of this region, as can also be visualized from PCA result (Figure 3D). Therefore, the coverage of population genetic diversity in $1 \mathrm{KG}$ is particularly insufficient in Southeast Asia.

\section{DISCUSSION}

In this study, we developed a method to quantitatively evaluate the genetic diversity coverage of $1 \mathrm{KG}$ based on PCA of genomewide SNP data in 140 populations worldwide. Our results showed insufficient representation of genetic diversity of some geographic regions in $1 \mathrm{KG}$, especially of Southeast Asia. Although our analysis showed that $1 \mathrm{KG}$ seemingly has reasonable coverage in many regions such as Africa, Europe and East Asia, we would like to point out that our results as well as conclusions might be conservative for the following reasons.

Firstly, our analysis relied on comparison of $1 \mathrm{KG}$ data and other available reference data such as HGDP and PASNP, which means that we could not give a good evaluation of $1 \mathrm{KG}$ data for their representation of genetic diversity if there were not sufficient data available in either $1 \mathrm{KG}$ or reference data. For example, there is no Central Asian data available in $1 \mathrm{KG}$ while there are data available in reference data from some Central Asian populations such as the Uyghur in both HGDP and PASNP. However, we did not draw a conclusion that $1 \mathrm{KG}$ lacks coverage of genetic diversity in Central Asia. Because previous studies reported that Central Asian populations such as the Uyghur are admixed populations with both Caucasian and East Asian ancestries (Xu and Jin, 2008; $\mathrm{Xu}$ et al., 2008, 2009), it is not clear whether the genetic diversity of Central Asian populations has already been represented jointly by European and East Asian populations since this relationship could not be revealed by PCA-based analysis. Regarding South Asia, the situation is similar. Since the phase II of $1 \mathrm{KG}$ has not yet released the data containing South Asian populations, we could not evaluate the genetic diversity coverage of $1 \mathrm{KG}$ data in South Asia in current study.

Secondly, our method was developed based on PCA results. On the one hand, population structure revealed by PCA is sensitive to rare variants but not common variants. We compared the minor allele frequency (MAF) spectrum of our data set and the MAF spectra of the data sets with similar number of random SNPs we created from $1 \mathrm{KG}$ data. As a result, our data set did not have excess of rare SNPs compared with the other randomly sampled data sets. On the other hand, PCA can only capture the majority of information in genome-wide data and populations, and fine-scale structures could not be fully revealed with limited dimensions of principal components, although the $D_{d}$ statistic can be easily extended to handle three-dimension or even higher dimensions PCs. Therefore, our analysis only evaluated whether $1 \mathrm{KG}$ data cover the majority of genetic diversity in a particular geographical region. From this aspect, our results may be conservative, i.e., genetic diversity coverage of $1 \mathrm{KG}$ data in those regions showing good representation could be overestimated.

Finally, we noted in our analysis that the sequence error using NGS existed in $1 \mathrm{KG}$, which could affect the estimation of population genetic diversity. Importantly, sequence errors tend to result in a higher estimation of genetic diversity. The consequence, as we discussed above, is overestimating the diversity coverage of $1 \mathrm{KG}$ data in those regions with genotyping data being compared. Therefore, regarding this issue, our results as well as conclusion could be conservative.

In summary, we concluded that the current version of $1 \mathrm{KG}$ data does not have sufficient genetic diversity coverage in Asia, especially in Southeast Asia that harbors significant cultural and linguistic diversity as well as the majority of genetic diversity in Asia. We thus suggest a good coverage of Asian populations, especially of Southeast Asian populations, be considered in $1 \mathrm{KG}$ or a regional effort be initialized to provide a more comprehensive characterization of the human genetic diversity in Asia, which is important for both evolutionary and medical studies in the future.

\section{ACKNOWLEDGMENTS}

These studies were supported by the National Science Foundation of China (NSFC) grants 31171218 and 30971577, by the Shanghai Rising-Star Program 11QA1407600, and by the Science Foundation of the Chinese Academy of Sciences (CAS; KSCX2-EW-Q-1-11; KSCX2-EW-R-01-05; KSCX2-EW-J-15-05). This research was supported in part by the Ministry of Science and Technology (MoST) International Cooperation Base of China. Shuhua $\mathrm{Xu}$ is Max-Planck Independent Research Group Leader and member of CAS Youth Innovation Promotion Association. Shuhua Xu also gratefully acknowledges the support of the National Program for Top-notch Young Innovative Talents and the support of K. C. Wong Education Foundation, Hong Kong. The funders had no role in study design, data collection and analysis, decision to publish, or preparation of the manuscript.

\section{AUTHOR CONTRIBUTIONS}

Shuhua $\mathrm{Xu}$ conceived and designed the study. Dongsheng Lu analyzed the data. Shuhua $\mathrm{Xu}$ and Dongsheng $\mathrm{Lu}$ wrote the paper. Shuhua Xu contributed to reagents, materials, and analysis tools. All authors have read and approved the final version of the manuscript. 


\section{REFERENCES}

Depristo, M. A., Banks, E., Poplin, P., Garimella, K. V., Maguire, J. R., Hartl, C., etal. (2011). A framework for variation discovery and genotyping using next-generation DNA sequencing data. Nat. Genet. 43, 491-498. doi: 10.1038/ng.806

Holm, H., Gudbjartsson, D. F., Sulem, P., Masson, G., Helgadottir, H. T., Zanon, C., et al. (2011). A rare variant in MYH6 is associated with high risk of sick sinus syndrome. Nat. Genet. 43, 316-320. doi: 10.1038/ ng.781

Li, J. Z., Absher, D. M., Tang, H., Southwick, A. M., Casto, A. M., Ramachandran, S., et al. (2008). Worldwide human relationships inferred from genome-wide patterns of variation. Science 319, 1100-1104. doi: 10.1126/science. 1153717

McKenna, A., Hanna, M., Banks, E., Sivachenko, A., Cibulskis, K., Kernytsky, A., etal. (2010). The Genome Analysis Toolkit: a MapReduce framework for analyzing nextgeneration DNA sequencing data. Genome Res. 20, 1297-1303. doi: $10.1101 /$ gr. 107524.110
Pasaniuc, B., Rohland, N., McLaren, P. J., Garimella, K., Zaitlen, N., Li, H., et al. (2012). Extremely low-coverage sequencing and imputation increases power for genome-wide association studies. Nat. Genet. 44, 631-635. doi: 10.1038/ng.2283

Price, A. L., Patterson, N. J., Plenge, R M., Weinblatt, M. E., Shadick, N. A. and Reich, D. (2006). Principal components analysis corrects for stratification in genome-wide association studies. Nat. Genet. 38, 904-909. doi $10.1038 / n g 1847$

Rosenberg, N. A., Pritchard, J. K., Weber, J. L., Cann, H. M., Kidd, K. K., Zhivotovsky, L. A., et al. (2002). Genetic structure of human populations. Science 298, 2381-2385. doi: 10.1126/science.1078311

Teo, Y. Y., Small, K. S., and Kwiatkowski, D. P. (2010). Methodological challenges of genome-wide association analysis in Africa. Nat. Rev. Genet. 11, 149-160. doi: 10.1038/ nrg2731

The 1000 Genomes Project Consortium. (2010). A map of human genome variation from populationscale sequencing. Nature 467, 10611073. doi: 10.1038/nature09534
The HUGO Pan-Asian SNP Consortium. (2009). Mapping human genetic diversity in Asia. Science 326, 1541-1545. doi: 10.1126/science. 1177074

The International HapMap Consortium. (2005). A haplotype map of the human genome. Nature 437, 12991320. doi: $10.1038 /$ nature 04226

$\mathrm{Xu}$, S., Huang, W., Qian, J., and Jin, L. (2008). Analysis of genomic admixture in Uyghur and its implication in mapping strategy. Am. J. Hum. Genet. 82, 883-894. doi: 10.1016/j.ajhg.2008.01.017

$\mathrm{Xu}, \quad$ S., and Jin, L. (2008). A genome-wide analysis of admixture in Uyghurs and a high-density admixture map for disease-gene discovery. Am. J. Hum. Genet. 83, 322336. doi: 10.1016/j.ajhg.2008.08.001

Xu, S., Jin, W., and Jin, L. (2009). Haplotype-sharing analysis showing Uyghurs are unlikely genetic donors. Mol. Biol. Evol. 26, 2197-206. doi: $10.1093 / \mathrm{molbev} / \mathrm{msp} 130$

Zawistowski, M., Gopalakrishnan, S. Ding, J., Li, Y., Grimm, S., and Zöllner, S. (2010). Extending rare-variant testing strategies: analysis of noncoding sequence and imputed genotypes.
Am. J. Hum. Genet. 87, 604-617. doi: 10.1016/j.ajhg.2010.10.012

Conflict of Interest Statement: The authors declare that the research was conducted in the absence of any commercial or financial relationships that could be construed as a potential conflict of interest.

Received: 01 April 2013; accepted: 14 June 2013; published online: 04 July 2013.

Citation: Lu D and Xu S (2013) Principal component analysis reveals the 1000 Genomes Project does not sufficiently cover the human genetic diversity in Asia. Front. Genet. 4:127. doi: 10.3389/fgene. 2013.00127

This article was submitted to Frontiers in Applied Genetic Epidemiology, a specialty of Frontiers in Genetics.

Copyright (c) $2013 \mathrm{Lu}$ and $\mathrm{Xu}$. This is an open-access article distributed under the terms of the Creative Commons Attribution License, which permits use, distribution and reproduction in other forums, provided the original authors and source are credited and subject to any copyright notices concerning any third-party graphics etc. 